\title{
Interactive comment on "Time series of Inland Surface Water Dataset in China (ISWDC) for 2000-2016 derived from MODIS archives” by S. Lu et al.
}

\section{Anonymous Referee \#1}

Received and published: 18 January 2019

\section{Manuscript:}

This manuscript showed some potential for publication. However, after looking into more detail it shows (1) a lot of duplication (previous paper Lu et a., 2017), (2) lack of sufficient accuracy assessment, (3) some incorrect quotes and very general statements. In terms of methodology this manuscript is almost exactly the same as presented by Lu et al., 2017. I do not disagree with the idea of using same methodology for a larger area of interest. But the methodological paragraphs are $90 \%$ identically with even same Figure and Table. Some adjustments are mentioned but are not discussed. In terms of accuracy assessment there is a lack of detailed analyses of the presented 
product. The presented numbers are based on calculated total areas over the entire region for maximum water surface area and do not even analyze the temporal scale. ESSDD The second dataset for cross comparison GWS (Pekel et al., 2016), was only used to compare visually for two zoom-in areas. With the final statement that both datasets look alike and have similar patterns. The temporal resolution is not validated at all. The effort of accuracy assessment which was performed is not state of the art and does not mirror the real quality of the presented product. In my view this manuscript does not have much of novelty and the statement about "lack of water surface products for China" is also not correct.

General Comments:

I would recommend English gramma check. Some sentences are understandable but have incorrect gramma (e.g. P1 L16, P1 L21, P 2 L5-6, P2 L17, P4 L2, P4 L10-11 and more) Please be consistent: either dataset or data set. Sometimes you use both options in one paragraph (e.g. P4) The methodological content is almost identical in many parts with Lu et al., 2017

Abstract and conclusion: Almost the same content!

Introduction

P 2 L 2: First statement -> please provide a source

P2 L 15: statement incorrect. The data is being produced from July 2002-ongoing.

P2 L19-24: this information could be summarized with key parameters in a table

P3 L3-4: NDWI is mostly known from McFeeters 1996 as well as Gao 1996

P3 L5: wrong citation of Feyisa et al. 2014 instead of 2018

P3 L7-8: incorrect statement for Pekel et al., 2014

P3 L24: LBV? 
P4 L2: first sentence does not make sense.

P4 L9-11: This statement is not true as there are datasets which reflects the spatial and temporal characteristics of surface water such as Pekel et al., 2016, Klein et al., 2017, Ji et al., 2018.

P4 L14: why does ISWDC ends with 2016?

Interactive

comment

P5 L16: Something should be following. Instead the paragraph ends here.

P5 Figure 1 is exactly the same as in Lu et al., 2017 if you have improved two steps, why don't update the figure as well and discuss the adjustments?

P5-7: is almost identically with your paper about Tibetan Plateau (TB). Even the table 1 with selected images is the same. Was there no difference of analyzing only TB and entire China? Maybe instead of copy-pasting the same text, you should clearly point out the updates and improvement which you did. At the moment, you only mention there were improvements but you do not mention where exactly these improvements are and why it was necessary.

Paragraph 3.2. boundary extraction? Your dataset is a raster data with pixel values for water and no water. For water boundary I would expect a vector dataset with lines or polygons which determines the boundaries of a water body. This paragraph is unclear and does not correspond to the header of the paragraph.

P7 L10-13: why is the difference between ice layer and water body in winter small?

\section{Accuracy Assessment}

P9 L7-8: what is the logic behind this procedure? You are comparing a static dataset of a certain year with the maximum area of ISWDC of corresponding year. What is the interpretation of calculated $\mathrm{R}^{2}$ in that case? To compare the total area over such a large area of all selected water bodies seems to be very shallow and not state of the art. I would expect a pixel based approach to actually assess the real quality at a 
certain time for a certain pixel.

Paragraph 4.2: it is only a visual interpretation of two zoom-in images without any ESSDD quantitative results.

5.1 The multi-year average analysis can also be done with GSW dataset. Therefore, I don't see any novelty or interesting facts especially since you mentioned that both datasets indicate similar patterns. Where is the advantage or novelty of using ISDWC in this case?

P13 Figure 5: this figure shows that, in general, the total surface water of China has a clear seasonality. However, the curves of single years cannot be determined due to figure design.

P13 Figure 6: In this Figure you show red lines, which are probably the water body borders. Are these polygons also distributed? So far I was only able to download binary raster data and no vector data.

P14 L11-12: again a statement which I do not agree with as the presented dataset covers almost the same time span as it is covered by GWS (Pekel et al., 2016).

Conclusion: is kept very general.

\section{Dataset}

The dataset itself is a mosaic for China area for temporal steps of 8 days and can used by interested scientists or organizations for different purposes. However, without sufficient quality layer or accuracy assessment especially of the different time steps.

Interactive comment on Earth Syst. Sci. Data Discuss., https://doi.org/10.5194/essd-2018-134, 2018. 